\title{
Ochrobactrum anthropi bacteremia in a preterm infant with cystic fibrosis
}

\author{
Fernando Gatti Menezes ${ }^{1}$, Maria Gabriela Ballalai Abreu ${ }^{1}$, Julia Yaeko Kawagoe ${ }^{1}$, \\ Arno Norberto Warth ${ }^{2}$, Alice D’Agostini Deutsch ${ }^{2}$, Maria Fernanda P S Dornaus ${ }^{2}$, \\ Marines Dalla Valle Martino ${ }^{3}$, Luci Correa ${ }^{1}$ \\ ${ }^{1}$ Serviço de Controle de Infecção Hospitalar, Hospital Albert Einstein, São Paulo, SP, Brazil. \\ ${ }^{2}$ Unidde de Terapia Intensiva Neonatal, Hospital Albert Einstein, São Paulo, SP, Brazil. \\ ${ }^{3}$ Laboratório de Microbiologia, Hospital Albert Einstein, São Paulo, SP, Brazil.
}

Submitted: January 13, 2013; Approved: September 9, 2013.

\begin{abstract}
Ochrobactrum anthropi infection in newborn patients is rare, and the treatment is challenging because of its widespread and unpredictable resistance to antimicrobial agents and discrepancies between in vitro susceptibility and in vivo efficacy. We report the clinical and microbiological characteristics of Ochrobactrum anthropi bacteremia in a preterm patient.
\end{abstract}

Key words: bacteremia, cystic fibrosis, newborn, meconium, premature birth.

Bacteremia after intestinal obstruction in a preterm patient is an alarming situation, and multiples microorganisms can cause this life-threatening infection, including gram-positive cocci, gram-negative rods, anaerobic species or yeasts. Therefore, empirical antimicrobial prescription therapy should cover a broad spectrum of pathogens, a combination of $\beta$-lactams, aminoglycosides, anaerobical and antifungal agents is commonly prescribed in many neonatal intensive care unit (NICU). However, there is a risk of translocation of opportunistic agents with multidrug resistance from intestinal flora to the bloodstream. Bowel resection may lead to bacteremia caused by microorganisms such as Ochrobactrum anthropi. We describe a case of blood stream infection (BSI) caused by Ochrobactrum anthropi in a preterm infant.

A 36-year-old woman was hospitalized in the 30th week of pregnancy because of decreased fetal movements. She gave birth to female preterm infant via cesarean delivery; her Apgar scores were 3 and 7 at 1 and $5 \mathrm{~min}$, respectively, and her birth weight was $1600 \mathrm{~g}$ (10-50th percentile). In the delivery room she showed apnea immediately after birth and endotracheal intubation was performed. The first physical examination revealed abdominal distension and pallor. On admission at NICU, the chest radiography showed multiple opacities in both lungs and one dose of surfactant was administrated. Ampicillin and amikacin was initiated empirically. Abdominal ultrasonography on her 1st day of life showed signs of intestinal obstruction with thick content compatible with midgut volvolus. The infant underwent laparotomy with bowel resection and metronidazole was added to antibiotic therapy. Total parental nutrition through peripheral venous access was initiated. Mechanical ventilation was suspended on 3rd day of life and on the day after she underwent a peripherally inserted central catheter (PICC). Ampicillin was discontinued after 7 days of treatment, amikacin and metronidazole were stopped after 10 days. After 2 days of minimal enteral feeding, on her 13th of life, the newborn began to show green gastric residuals through her gastric tube. After contrast enema, abdominal obstruction was diagnosed again. After three days, cystic fibrosis was investigated and confirmed through molecular biology (heterozygosis for G542X and W1282X mutation). On the following day another laparotomy with bowel resection was performed due to obstruction. Culture specimens were not collected during the surgery. Blood cultures were obtained after the procedure and the combination of ampicillin, amikacin and metronidazole was re-initiated. After four days, microbial growth was detected by BACTEC system. First of all, automated microbial identification was performed using Vitek 2 system and then Vitek MS that uses innovative mass spectrometry technology (MALDI-TOF). Both instruments diagnosed 
Ochrobactrum anthropi. Then the identification was confirmed by PCR and sequencing of the 16S ribosomal RNA genes (molecular biology). Antimicrobial susceptibility testing was performed by Etest on Mueller Hinton agar plates. The interpretative criteria for antimicrobial susceptibility testing were according to the Clinical Laboratory Standards Institute document. The isolate was susceptible to meropenem (minimum inhibitory concentration equal $0.38 \mathrm{mcg} / \mathrm{mL}$ ), cotrimoxazole (minimum inhibitory concentration equal $0.032 \mathrm{mcg} / \mathrm{mL}$ ), amikacin (minimum inhibitory concentration equal $8 \mathrm{mcg} / \mathrm{mL}$ ), and resistant to ceftazidime (minimum inhibitory concentration > $256 \mathrm{mcg} / \mathrm{mL}$ ). So, ampicilin and metronidazole were discontinued and meropenem was added to amikacin. Despite appropriate therapy, four peripherally obtained blood cultures bottles continued to grow Ochrobactrum anthropi. The initial PICC was removed on the day that the last blood culture showed growth and another PICC was inserted. The catheter tip culture showed no growth. Amikacin and meropenem were discontinued at 21 and 25 days, respectively. After that, the infant underwent two other abdominal surgical procedures (ileostomy and ileostomy closure).

Ochrobactrum anthropi, previously classified as Achromobacter spp or Centers for Disease Control groups $\mathrm{Vd}$, is a Gram-negative, nonfastidious, motile, aerobic bacillus, non-lactose-fermenting that oxidizes glucose, ubiquitous in the environment. Besides the difficulty of its microbiological characterization, this pathogen is considered to be an opportunistic pathogen with low pathogenicity often causing infection in severely ill or immunocompromised patients. The most human cases reported have been associated with indwelling medical devices, such as catheter venous central, drainage tubes, and intraperitoneal catheters. The ability of the pathogen to adhere to silicone, which is similar to that of Staphylococcus aureus and Staphylococcus epidermidis, has been demonstrated in vitro and may play a role in catheter associated infections (Duran et al., 2009; Yagüe-Muñoz et al., 2010). It can be speculated that the catheter might have been a source of intravascular contamination with this agent.

Although this pathogen is part of the normal flora of the large intestine, it might have been another source of bloodstream infection after two intestinal surgeries. Intestinal obstruction is considered a common complication in cystic fibrosis due to an up-regulation of the epithelium sodium channel which enhances sodium and fluid absorption from the luminal mucus. This situation predisposes to intestinal obstruction and leads to stasis, thus favoring the growth of bacteria, and may allow bacteria translocation into the bloodstream (Colombo et al., 2011; Wi et al., 2010; Yagüe-Muñoz et al., 2010).

Ochrobactrum anthropi isolates are typically susceptible to aminoglycosides, carbapenems, cotrimoxazole, quinolones, and sulfonamides; are variably susceptible to rifampicin and tetracyclines; and are resistant to $\beta$-lactams (other than carbapenems), chloramphenicol, and macrolides. Chromosomal class $\mathrm{C}$ b-lactamase may be one reason for the extensive resistance of this bacterium to $\beta$-lactams (Thoma et al., 2009). The strain of the present case showed a susceptibility pattern with resistance to ceftazidime and susceptibility to meropenem, amikacin and cotrimoxazole.

The optimal therapeutic approach to patients with Ochrobactrum anthropi bacteremia remains uncertain. However, the poor correlation of in vitro susceptibility data associated with the intrinsic antimicrobial resistance of this microorganism emphasizes the prescription of antibiotic combination. Better outcomes have been reported using combinations of aminoglycosides, quinolones and carbapenem or cotrimoxazole. In each case, the identification of the microorganism must be confirmed by reliable methods and the antibiogram should be executed (Kettaneh et al., 2003; Vaidya et al., 2006; Aly et al., 2007; Chiang et al., 2009).

This case highlights the awareness of the potential role of Ochrobactrum anthropi in causing BSI after meconium ileus, the intestinal manipulation in multiple surgeries, and the microbiological characterization challenges. While the optimal choice among such agents for the treatment of these infections is likewise unknown, the clinician should note that Ochrobactrum anthropi isolate appears to be resistant to many antibiotics commonly employed in the empirical treatment in the NICU. Therefore, the antibiotic combination to broad-spectrum coverage appears to be a better choice. Further clinical experiences and epidemiologic studies with invasive diseases caused by Ochrobactrum anthropi need to be published in order to be able to more fully evaluate its pathogenesis and treatment.

\section{Acknowledgments}

We thank Jacyr Pasternak, PhD, MD that provided general support.

\section{References}

Aly NYA, Salmeen HN, Joshi RM (2007) Ochrobactrum anthropi Bacteremia in a Child with Inborn Error of Mitochondrial Fatty Acid Oxidation. Med Prin Pract 16:463-465.

Chiang CC, Tsai YY, Lin JM, Chen WL (2009) Chronic endophthalmitis after catarata surgery secondary to Ochrobactrum anthropi. Eye(Lond) 23:1237-1238.

Colombo C, Ellemunter H, Houwen R, Munck A, Taylor C, Wilschanski M, ECFS (2011) Guidelines for the diagnosis and management of distal intestinal obstruction syndrome in cystic fibrosis patients. J Cyst Fibros 10:24-28.

Duran R, Vatansever Ü, Acunas B, Basaran ÜN (2009) Ochrobactrum anthropi bacteremia in a preterm infant with meconium peritonitis. Int J Infect Dis 13:61-63.

Kettaneh A, Weill FX, Poilane I, Fain O, Thomas M, Herrmann JL, Hocqueloux L (2003) Septic Shock Caused by Ochrobactrum anthropi in an Otherwise Healthy Host. J Clin Microbiol 41:1339-1341. 
Thoma B, Straube E, Scholz HC, Al Dahouk S, Zöller L, Pfeffer M, Neubauer H, Tomaso H (2009) Identification and antimicrobial susceptibilities of Ochrobactrum spp. Int J Med Microbiol 299:209-220.

Vaidya SA, Citron DM, Fine MB, Murakami G, Goldstein EJ (2006) Pelvic Abscess Due to Ochrobactrum anthropi in an Immunocompetent Host: Case Report and Review of the Literature. J Clin Microbiol 44:1184-1186.

Wi YM, Peck KR (2010) Biliary Sepsis Caused by Ochrobactrum anthropi. Jpn J Infect Dis 63:444-446.
Wi YM, Soh KM, Rhee JY, Oh WS, Peck KR, Lee NY, Song JH (2007) Spontaneous Bacterial Peritonitis due to Ochrobactrum anthropi: A Case Report. J Korean Med Sci 22:377-379.

Yagüe-Muñoz A, Gregori-Roig P, Valls-López S, Pantoja-Martínez J (2010) Ochrobactrum anthropi bacteremia in a child with cystic fibrosis. Enferm Infecc Microbiol Clin 28:137138.

All the content of the journal, except where otherwise noted, is licensed under a Creative Commons License CC BY-NC. 